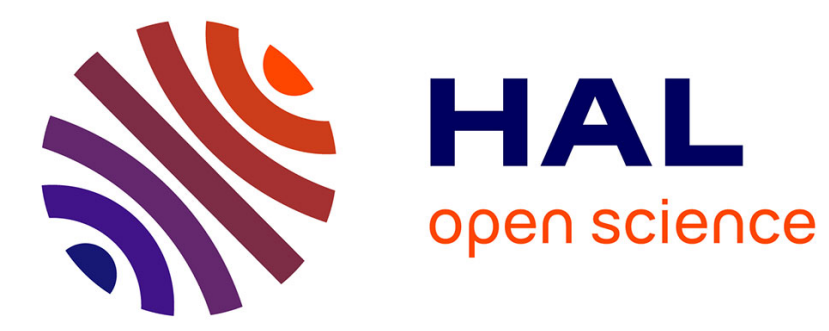

\title{
Deformation of a liquid near an AFM tip: molecular dynamics approach
}

Lolita Hilaire, Bertrand Siboulet, Sophie Charton, René Ledesma-Alonso, Dominique Legendre, Philippe Tordjeman, Jean-François Dufrêche

\section{To cite this version:}

Lolita Hilaire, Bertrand Siboulet, Sophie Charton, René Ledesma-Alonso, Dominique Legendre, et al.. Deformation of a liquid near an AFM tip: molecular dynamics approach. Langmuir, 2020, 36 (30), pp.8993-9004. 10.1021/acs.langmuir.0c00023 . hal-02924910

\section{HAL Id: hal-02924910 https://hal.umontpellier.fr/hal-02924910}

Submitted on 10 Feb 2022

HAL is a multi-disciplinary open access archive for the deposit and dissemination of scientific research documents, whether they are published or not. The documents may come from teaching and research institutions in France or abroad, or from public or private research centers.
L'archive ouverte pluridisciplinaire HAL, est destinée au dépôt et à la diffusion de documents scientifiques de niveau recherche, publiés ou non, émanant des établissements d'enseignement et de recherche français ou étrangers, des laboratoires publics ou privés. 


\title{
Deformation of a Liquid Near an AFM Tip: Molecular Dynamics Approach
}

\author{
Lolita Hilaire, $^{\dagger}$ Bertrand Siboulet, $^{\ddagger}$ Sophie Charton, $^{\dagger}$ René Ledesma-Alonso, $^{\natural}$ \\ Dominique Legendre, Philippe Tordjeman, " and Jean-François Dufrêche*, \\ $\dagger C E A$, DEN, DMRC, Univ Montpellier, Marcoule, France \\ $\ddagger I C S M, C E A, C N R S$, Univ Montpellier, ENSCM, Marcoule, France
}

ๆInstitut de Mécanique des Fluides de Toulouse (IMFT), INPT-CNRS, Université de Toulouse, Allée du Professeur Camille Soula, 31400 Toulouse, France

E-mail: jean-francois.dufreche@icsm.fr

\begin{abstract}
The interaction between an AFM probe and a thin water film deposited over a flat substrate is studied using Molecular Dynamics (MD). The effects of the film thickness and of the probe radius on both the deformation height of the liquid interface, and the distance of the jump to contact at which the liquid comes in direct contact with the probe, are investigated. The dynamics of the surface deformation and the role of interface fluctuations are studied in details. The systems considered belong to the thin film regime described in a semi-analytical model previously established by Ledesma et al. (Langmuir 2013, 29, 7749-7757). MD simulations predict that for shallow films, both the distance at which the jump to contact occurs, and the surface maximal deformation height increase steadily with the layer thickness regardless of the probe radius, which is in agreement with the theoretical model proposed. The results also reveal that the deformation of the interface is localized under the probe's apex, and that it is
\end{abstract}


highly subject to fluctuations which result in an instability of the deformation for the considered systems. The molecular approach hence points out the importance of fluctuations when it comes to the description of nanosystems involving soft interfaces. This unveils the needs for the establishment of thermal models to improve accuracy.
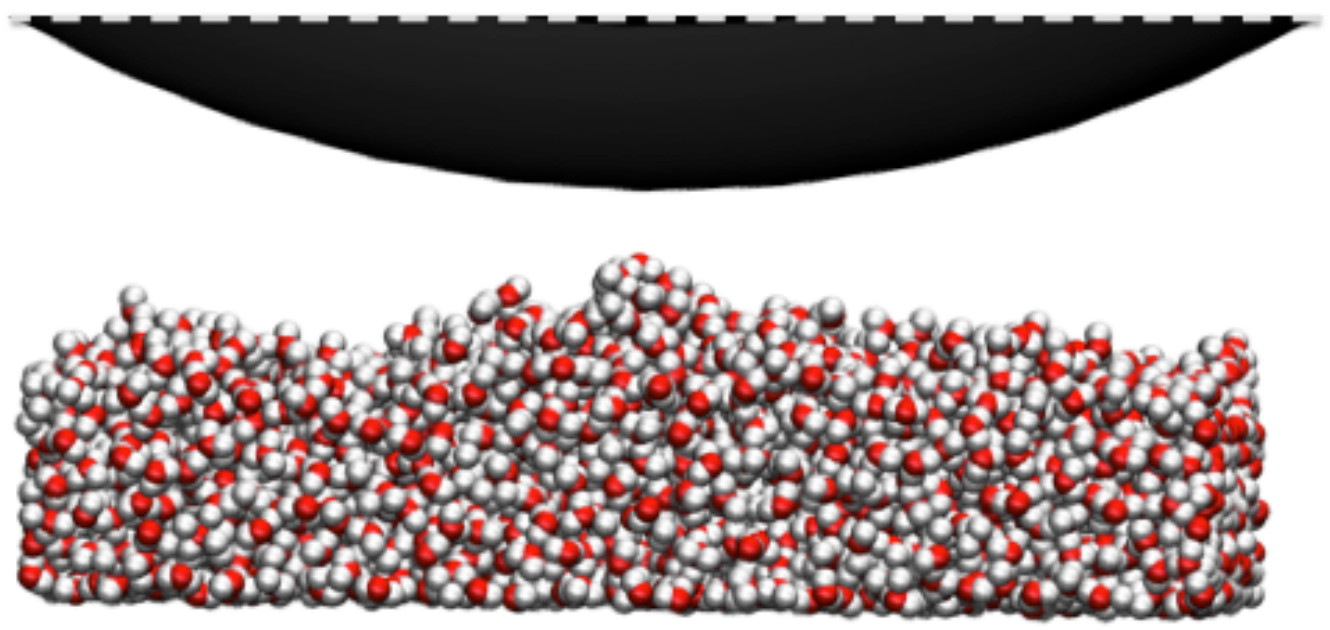

Figure 1: Snapshot of surface deformation for a liquid water film of thickness $E=2.2 \mathrm{~nm}$ in vacuum interacting with an AFM probe of radius $R=10 \mathrm{~nm}$.

\section{Introduction}

Since 1986, Atomic Force Microscopy (AFM) has enabled to probe material properties such as elasticity, Hamaker constants and adhesion ${ }^{1}$. AFM measurements achieved up to a molecular resolution and can measure surface forces as low as $10^{-12} \mathrm{~N}^{2}$. The development of this tool has considerably accelerated the studies of surface topography ${ }^{345}$ and the characterization of liquid samples ${ }^{678}$. The AFM probe is attached to a cantilever. The positions of the probe and of the cantilever vary in response to a change in the sample's surface topography. However when the distance between the apex of the probe and the sample surface gets too short and reaches a critical value, a jump-to-contact phenomenon in which the sample adheres to the probe, occurs ${ }^{91011}$. This phenomenon is more pronounced in the case of soft samples when surface deformation is observed ${ }^{121314}$. 
In most situations, the wetting of the probe or its adhesion on the sample have to be avoided in AFM measurements, it is therefore necessary to understand and describe both the surface deformation and the jump to contact. Some models have been proposed to describe this phenomenon ${ }^{1516}$ but they may lack adequacy at the lowest scale. Indeed, at a molecular scale, a particle's environment resembles more a discrete set of particles than a continuous medium ${ }^{17}$. Properties considered constant in continuous models may become heterogeneous, leading to a behavior of the matter that deviates from the theory. Thanks to the emergence of powerful computational facilities, the modelling of nano-systems has become possible ${ }^{18}$ thus allowing to verify and adjust theoretical models. Molecular Dynamics (MD) is a one of the most appropriate tool used for this purpose. Furthermore, its relevance in the description of the jump-to-contact phenomenon has already been pointed out ${ }^{19}$.

This aim of this work is to provide a better understanding of the jump-to-contact phenomenon in the case of very thin films which are difficult to study experimentally. The atomistic vision provided by MD is fully relevant to study nano-systems. A molecular approach is therefore well suited to investigate the behavior of thin liquid films in AFM experiments. In this study, systems composed of AFM probes of various radii in interaction with liquid water films of various thicknesses, deposited over a flat substrate are modelled. The interface deformation and the jump to contact have been monitored through the simulation of the probe approach.

In the following, the radial surface deformation is noted $\eta(r)$, and the distance between the centre of the probe and the undistorted surface is noted $D$. The gap between the apex deformation and the surface of the probe in $r=0$ is noted $\epsilon$ (see Figure 2). $D_{\min }$ is the separation distance where the jump to contact occurs, $\eta_{\max }$ the corresponding maximum deformation of the surface in $r=0$, and $\epsilon_{\min }$ the minimum gap between the deformed surface 


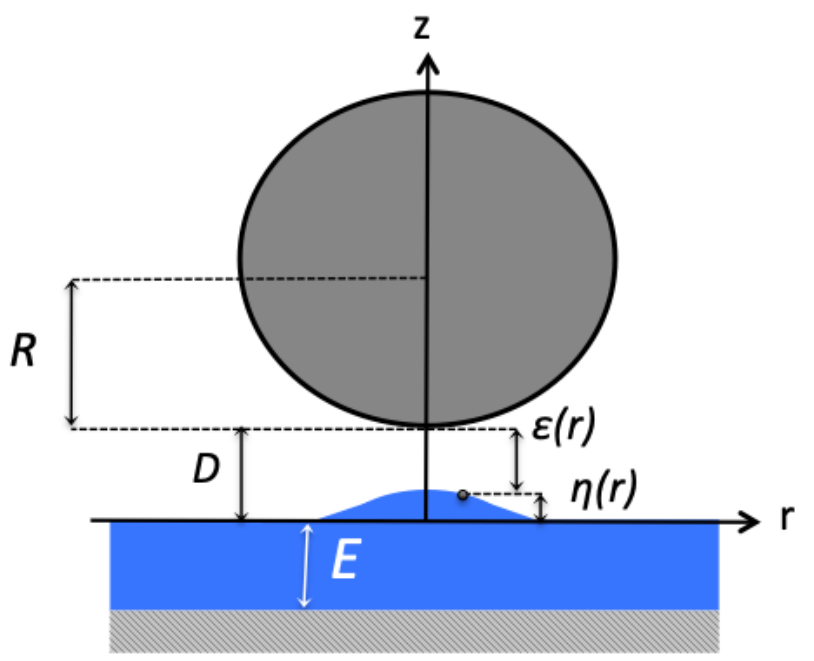

Figure 2: Interaction between an AFM probe and a liquid film deposited over a flat substrate.

and the surface of the probe. The previously defined parameters are related by:

$$
D_{\min }=R+\eta_{\max }+\epsilon_{\min } .
$$

In a first part, the continuous model describing the deformation of the surface along with the capillary wave theory describing the fluctuations of the interface, are detailed. The strategy adopted and the parametrical study carried to test the semi-analytical model at the nano-scale is then presented. Also, because fluctuations have been shown to have an important contribution in AFM measurements ${ }^{20}$, a last part is devoted to the detailed analysis of the surface deformation and the rate of interface oscillations.

\section{Theoretical approach}

\section{Continuous Model $^{21}$}

The model proposed by Ledesma et al. ${ }^{21}$ to describe the surface deformation is applied to a system composed of an AFM probe in interaction with a water film deposited over a flat 
substrate. Dispersion forces between the probe and the sample may cause a deformation of the soft surface ${ }^{1}$. The deformation can be quantitatively determined via generalized Laplace equation which expresses the pressure difference across a curved interface while accounting for the effects of dispersion forces, surface tension, and gravity. Dispersion forces in the system occur between the probe and the liquid, and between the liquid and the substrate. The excess term corresponds to the potential energy difference between the perturbed and the unpertubed state. The expression of the interaction energy between two spheres of radius $R_{1}$ and $R_{2}$, and particle density of $\rho_{1}$ and $\rho_{2}$, whose centres are separated by a distance $C$ is given by ${ }^{22}$ :

$$
U=-\frac{\pi^{2} \rho_{1} \rho_{2} \lambda}{6}\left[\frac{2 R_{1} R_{2}}{C^{2}-\left(R_{1}+R_{2}\right)^{2}}+\frac{2 R_{1} R_{2}}{C^{2}-\left(R_{1}-R_{2}\right)^{2}}+\ln \left(\frac{C^{2}-\left(R_{1}+R_{2}\right)^{2}}{C^{2}-\left(R_{1}-R_{2}\right)^{2}}\right)\right],
$$

where $\lambda$ is the London-Van der Waals constant.

In the considered system, the interaction energy between the probe and a point in the liquid is obtained from equation (2). To this end, the probe is modelled as a sphere of radius $R_{1}=R$ and the point of the liquid as a sphere of radius $R_{2} \rightarrow 0$. The corresponding pressure is in energy per unit of volume:

$$
P_{p l}=-\frac{4 \lambda \pi \rho_{1} \rho_{2} R^{3}}{3} \frac{1}{\left[(D-\eta)^{2}+r^{2}-R^{2}\right]^{3}} .
$$

Including the Hamaker constant of the probe over the liquid, $H_{p l}=\pi^{2} \lambda \rho_{1} \rho_{2}$, and neglecting the energy between the probe and the non deformed liquid interface, i.e. $P_{p l}^{\infty}=0$, the excess pressure applied by the probe over the liquid $\Pi_{p l}$ can be defined as:

$$
\Pi_{p l}=P_{p l}-P_{p l}^{\infty}=-\frac{4 H_{p l} R^{3}}{3 \pi\left[(D-\eta)^{2}+r^{2}-R^{2}\right]^{3}} .
$$

Similarly the interaction energy between the substrate and a point in the liquid can be obtained from equation (2) when the surface is considered as a sphere of infinite radius, i.e. 
$R_{2} \rightarrow+\infty$. Using the system parameters and the Hamaker constant between the substrate and the liquid $H_{l s}$, the following pressure is obtained:

$$
P_{l s}=-\frac{H_{l s}}{6 \pi}\left[\frac{1}{(E+\eta)^{3}}\right] .
$$

The expression for the undistorted state is given by:

$$
P_{l s}^{\infty}=-\frac{H_{l s}}{6 \pi E^{3}} .
$$

Hence, the expression of the excess pressure $\Pi_{l s}$ applied over the liquid by the substrate reads:

$$
\Pi_{l s}=P_{l s}-P_{l s}^{\infty}=-\frac{H_{l s}}{6 \pi}\left[\frac{1}{(E+\eta)^{3}}-\frac{1}{(E)^{3}}\right] .
$$

Finally, considering the excess pressure due to gravity, the final modified Laplace equation is obtained:

$$
2 \kappa \gamma=\Pi_{p l}-\Pi_{l s}-\Delta \rho g \eta,
$$

where $\kappa$ is the deformation curvature, $g$ is the standard gravity, and $\gamma$ the surface tension.

Three distinct regimes can be reached depending on the considered liquid thickness $E^{21}$. These tendencies are delimited by two characteristic film thicknesses, $E_{\gamma}$ and $E_{g}$, related to the characteristic lengths $\lambda_{C}$ and $\lambda_{F}$. The capillary length $\lambda_{C}$ is the ratio of surface tension and the gravitational forces, whereas the characteristic film thickness length $\lambda_{F}$ is the ratio of the surface tension and the Hamaker's forces. They are respectively defined as:

$$
\lambda_{C}=\left(\frac{\gamma}{\Delta \rho g}\right)^{1 / 2}, \quad \lambda_{F}=\left(\frac{2 \pi \gamma E^{4}}{H_{l s}}\right)^{1 / 2} .
$$

The expressions of $E_{\gamma}$ and $E_{g}$ proposed by the semi-analytical model are ${ }^{21}$ :

$$
E_{\gamma}=\left(\frac{R^{2} H_{l s}}{2 \pi \gamma}\right)^{1 / 4}, \quad E_{g}=\left(\frac{H_{l s}}{2 \pi \Delta \rho g}\right)^{1 / 4} .
$$

The values of the characteristic lengths and characteristic film thicknesses for each modeled 
systems are reported in Table 2.

Ledesma et al. ${ }^{21}$ established the following tendencies:

- Thin film regime is obtained for $E<E_{\gamma}$. In this regime $\lambda_{F}<<\lambda_{C}$, the interaction of the substrate is dominant. The liquid behaves as a tiny drop or a thin film, depending on the type of wetting. The effects of the disjoining pressure are important ${ }^{23}$. The action of the substrate over the interface dominates and balances the action exerted by the tip. $D_{\min }$ and $\eta_{\max }$ are independent of the value of tip radius but depend on the film thickness. The deformation of the interface is limited to a narrow zone, and exhibits high curvature.

- Bulk liquid regime is obtained for $E>E_{g}$. In this regime $\lambda_{F}>>\lambda_{C}$, gravitational forces are dominant and tend to flatten the surface. The interface is no longer subject to the action of the substrate, deformation is less localized and of reduced curvature. Both $\eta_{\max }$ and $D_{\min }$ depend on the tip radius and are independent of the film thickness.

- For $E_{\gamma}<E<E_{g}$, an intermediate behaviour is predicted. $\eta_{\max }$ and $D_{\min }$ are increasing functions of the film thickness and both tend towards a plateau value dependant on the tip radius.

AFM experiments were carried to test the model. Results showed good agreement with the theory for the bulk and intermediate regimes ${ }^{24}$. The investigation of the thin film regime was however limited, due to difficulties in carrying AFM measurements on films having thicknesses of only a few nanometres. The aim of this work is to provide a better description of the thin film regime. In this aim, a parametric study was performed to evaluate the influence of the probe radius $R$ and the film thickness $E$ on the maximum deformation height $\eta_{\max }$ and the jump to contact distance $D_{\min }$. This will determine if the modelled systems follow the thin film regime tendency. The deformation profiles obtained from MD 
simulations are then compared to those predicted by the continuous model, and the role of thermal fluctuations is evaluated. This will enable to access the accuracy of the continuous model at the molecular scale.

\section{Fluctuations of a liquid interface}

For very thin films as considered in this work, the fluctuations of the surface cannot be neglected because they have a strong influence on the instant film thickness. To quantify the effects of those oscillations, the capillary wave theory was adopted. This theory states that the oscillation of the interface results from the competition between the thermal agitation, that tends to increase the surface area, and the surface tension that acts to reduce it. The free energy associated to the fluctuations is expressed by ${ }^{23}$ :

$$
H_{\text {cap }}=\gamma \iint \mathrm{d}^{2} A
$$

where $\mathrm{d}^{2} A$, the change in surface area, includes a non-planarity correction. The resulting deformation field can be expressed in Fourier's space where capillary waves are represented by a sum of modes characterized by a wave number $q$. Using a Fourier analysis and summing all the different modes, the standard deviation of the surface fluctuations reads:

$$
\sigma_{\text {cap }}=\sqrt{\frac{k_{\mathrm{B}} T}{2 \pi \gamma} \ln \left(\frac{q_{\mathrm{max}}}{q_{\mathrm{min}}}\right)}
$$

where $k_{\mathrm{B}}$ is the Bolzmann constant, $T$ the temperature, $q_{\max }=2 \pi / \xi$ where $\xi$ is a typical molecular size chosen to be the average diametre of a water molecule $\xi=0.3 \mathrm{~nm}$, and $q_{\text {min }}=2 \pi / L$ where $L$ is the lateral dimension of the box whose surface is $L \times L$, here $L=8.0 \mathrm{~nm}$. This results in an amplitude of fluctuation of $\sigma_{\text {cap }}=1.9 \times 10^{-1} \mathrm{~nm}$. The amplitude of the fluctuations depends on the choices made for $q_{\max }$. However, because of the logarithm, the amplitude of fluctuations are not strongly affected by changes in the 
wavelengths boundaries.

The standard deviation of the surface fluctuations was also quantified numerically by the mean of MD simulations, to assess the validity of this description at a molecular scale and its possible application to a distorded interface. This work focusing on very thin films deposited over a flat substrate, we also modelled the changes of fluctuations ${ }^{25}$ due to the substrate presence.

\section{Molecular Dynamics strategy and method}

\section{Simulations premises}

It has been previously demonstrated thanks to MD simulations that Laplace equation remains valid at nano-scale ${ }^{26}$. In the present study, MD simulations are carried out to model the surface deformation and the jump to contact using the MD code LAMMPS ${ }^{27}$. The simulation domain illustrated in Figure 2 consists in an AFM probe of radius $R$ and a liquid film of initial thickness $E$. The centre of the probe is at a distance $D$ of the undistorted liquid surface. The liquid is deposited on a wall acting as a substrate. In the MD simulations, this wall is modelled by a fictive surface with a given potential.

The liquid film is modelled with an extended simple point charge (SPC/E) water model. In this model, the oxygen atoms are assumed to be charged Lennard-Jones (LJ) particles and the hydrogen atoms as charged particles but without LJ interactions ${ }^{28}$. The SPC/E model imposes constraints on the $\mathrm{O}-\mathrm{H}$ bond length and on the value of the molecule's angle.

This constraints were applied with the SHAKE algorithm ${ }^{29}$. The corresponding potential energy $U_{i j}$ between two atoms $i$ and $j$ separated by $r_{i j}$ is a $12 / 6 \mathrm{LJ}$ potential associated with 
a Coulumbic interaction:

$$
U_{i j}(r)=4 \epsilon\left[\left(\frac{\sigma}{r}\right)^{12}-\left(\frac{\sigma}{r}\right)^{6}\right]+\sum_{i, j} \frac{q_{i} q_{j}}{r_{i, j}}
$$

where $(\epsilon, \sigma)$ are the LJ coefficients of the corresponding atoms. To speed up the calculation, a cut-off radius $r_{c}$, classical tool in MD, is attributed to the potential. Above $r_{c}$ the LJ potential is neglected. The potential is shifted to ensure that its value in $r_{c}$ is 0 . The Coulumbian term is calculated by the P3M (particle-particle-particle mesh) method.

The substrate is modelled as an uncharged wall to which is attributed a 9/3 LJ potential, which corresponds to the LJ potential of a plane:

$$
U(z)=\epsilon_{\text {sub }}\left[\frac{2}{15}\left(\frac{\sigma_{\text {sub }}}{z}\right)^{9}-\left(\frac{\sigma_{\text {sub }}}{z}\right)^{3}\right]
$$

A cut-off $z_{c}$ is attributed to the potential over the $z$-axis. The Lennard-Jones coefficients $\left(\epsilon_{\text {sub }}, \sigma_{\text {sub }}\right)$ are chosen in order to fit the potential of the substrate over the liquid described by equation (7).

The AFM probe is modelled as an uncharged sphere of radius $R$ with the following potential:

$$
U(r)=\frac{4 H_{p l} R^{3} M_{H_{2} O}}{3 \rho_{H_{2} O} \pi\left(r^{2}-(r+R)^{2}\right)^{3}}+\frac{A}{r^{12}},
$$

where $H_{p l}$ is the Hamaker constant of the probe over the water, $\rho_{\mathrm{H}_{2} \mathrm{O}}$ and $M_{\mathrm{H}_{2} \mathrm{O}}$ the density and molecular mass of water respectively. The value of this potential is obtained by applying equation (4) to water, to which is added a repulsive component in which $A=1 \mathrm{kcal} \cdot \mathrm{mol}^{-1} \cdot \AA^{12}$. A cut-off $r_{c}$ is also attributed to the potential. $r_{c}$ and $z_{c}$ are chosen equal and as large as possible to ensure that the probe interacts with all of the liquid film. The probe/fluid potential has been implemented in the MD code in a tabular way.

The numerical value of the potential parameters are given in Table 1 . The Hamaker con- 
stants were chosen in agreement with the material used in AFM components, more specifically the values of $H_{p l}$ and $H_{l s}$ correspond respectively to a silicon probe, and to a silicon substrate.

Table 1: Numerical values of parameters used for the different potentials

\begin{tabular}{lccc}
\hline Element & $\epsilon(\mathrm{eV})$ & $\sigma(\AA)$ & $q(\mathrm{e})$ \\
\hline $\mathrm{O}_{w}{ }^{30}$ & $6.76208 \times 10^{-3}$ & 3.16556 & -0.8476 \\
$\mathrm{H}_{w}{ }^{30}$ & 0.00000 & 0.00000 & 0.4238 \\
Substrate & 0.01000 & 3.00000 & 0.0000 \\
\hline \multicolumn{2}{c}{ Hamaker constants } & \multicolumn{2}{c}{ Cut-off values } \\
\hline$H_{p l}(\mathrm{~J})^{21}$ & $4.10^{-20}$ & $z_{c}(\mathrm{~nm})$ & 2,5 \\
$H_{l s}(\mathrm{~J})^{21}$ & $4.10^{-20}$ & $r_{c}(\mathrm{~nm})$ & 2,5 \\
\hline
\end{tabular}

All simulations were carried in the NVT ensemble at $300 \mathrm{~K}$. Due to the small size of the simulation domain, evaporation effect were ignored ${ }^{31}$. The timestep chosen was the femtosecond. In all the simulations, the probe was approaching the liquid surface at the speed of $0.01 \mathrm{~nm}$ each ps until the jump to contact occurs or surface deformation is observed.

\section{Parametric study}

The parametric study aims at evaluating the influence of the probe radius $R$ and the film thickness $E$ on the values of the jump-to-contact threshold distance $D_{\min }$ and the maximum deformation height $\eta_{\max }$. To this end, several systems were constructed in a simulation box of $5.704 \times 5.704 \times 8.0 \mathrm{~nm}^{3}$. Table 2 lists all modelled systems and their characteristics.

The tip radius is defined in the potential attributed to the probe, the change of radius is made by changing the potential value. There is therefore no constraint on the radius choice, the values are however taken in order to correspond with real AFM tip radii. The liquid film requires to be modelled atom by atom. Hence, due to the very long calculation 
time of large systems, only relatively thin films have been considered. In comparison to the different regimes described by Ledesma et al. in the semi-analytical model ${ }^{21}$, the simulation domains belong to the thin film regime or the beginning of the intermediate regime. The characteristic thickness delimiting the end of the thin film regime $E_{\gamma}$ and the corresponding characteristic length $\lambda_{F}$ were evaluated for each system, and are reported in Table 2 . The same values of the characteristic thickness delimiting the beginning of the bulk regime $E_{g}$ and the capillary length $\lambda_{C}$ were considered for all systems and take the values: $E_{g}=900 \mathrm{~nm}$ and $\lambda_{C}=8.07 \mathrm{~mm}$.

Table 2: Parameters and characteristic thicknesses of the different modelled systems

\begin{tabular}{cccccc}
\hline$R(\mathrm{~nm})$ & $E(\mathrm{~nm})$ & $N_{\mathrm{H}_{2} \mathrm{O}}$ & $E_{\gamma}(\mathrm{nm})$ & $\lambda_{F}(\mathrm{~nm})$ & Regime \\
\hline 10 & 1.1 & 1041 & 1.7 & 24.2 & Shallow film \\
10 & 1.3 & 1312 & 1.7 & 33.8 & Shallow film \\
10 & 1.6 & 1595 & 1.7 & 51.2 & Shallow film \\
10 & 1.8 & 1825 & 1.7 & 64.8 & Intermediary \\
10 & 2.0 & 2016 & 1.7 & 80.0 & Intermediary \\
10 & 2.2 & 2055 & 1.7 & 96.8 & Intermediary \\
1 & 2.2 & 2055 & 0.5 & 96.8 & Shallow film \\
25 & 2.2 & 2055 & 2.7 & 96.8 & Shallow film \\
50 & 2.2 & 2055 & 3.8 & 96.8 & Shallow film \\
\hline
\end{tabular}

$R$ : Probe radius; $E$ : Film thickness; $N_{\mathrm{H}_{2} \mathrm{O}}$ : Number of water molecules in the film; $E_{\gamma}$ : Shallow film regime characteristic thickness; $\lambda_{F}$ : Characteristic film thickness length;

The influence of the film thickness $E$ and the probe radius $R$ on $D_{\min }$ and $\eta_{\max }$ was investigated in two steps. On the first step, simulations are carried at different film thicknesses for a constant tip radius. On the second step, simulations are carried at a different probe radii for a constant film thickness. The aim is to obtain the critical distance $D_{\text {min }}$ of the jump to contact and the corresponding maximum interface deformation $\eta_{\max }$ for each system.

As the threshold distance $D_{\min }$ is dependant on the tip radius $R$, we considered the value of $D_{\min }-\mathrm{R}$ for comparison purposes. This relative distance allows for the comparison of 
results obtained at different film thicknesses and different tip radii. The maximum height deformation $\eta_{\max }$ however, does not include the value of the tip radius it is therefore studied directly.

In this configuration, the interface is defined by the 98 oxygen atoms having the higher position over the $z$-axis. The reference value of the non-deformed interface is calculated at the beginning of each simulation. The positions of the tip and of the atoms describing the interface are exported every 0.5 ps, i.e. each 500 timesteps.

\section{Deformation profiles}

Besides the parametric study, the interface deformation profiles have been studied in further details. The deformation obtained in the MD simulations were compared to the deformation predicted by the continuous model ${ }^{21}$. A relaxation time $\tau$ of the system is introduced ${ }^{14}$ :

$$
\tau=\frac{R \mu}{\gamma},
$$

where $\mu$ is the viscosity and $\gamma$ the surface tension of the considered liquid (for SPC/E water at $300 \mathrm{~K}, \mu=8.2 \times 10^{-4}$ Pa.s ${ }^{32}$ and $\gamma=63.2 \mathrm{mN} / \mathrm{m}^{33}$ ). In the considered range of probe radii, the relaxation time is expected to be in $\tau \in\left[10^{-10}, 10^{-9}\right] \mathrm{s}$. Simulations show, however, that a few hundreds picoseconds are sufficient to observe a deformation. This deformation never reaches a steady state for it is constantly oscillating due to thermal fluctuations.

Deformation profiles studied by MD need to be averaged over time to smoothen the effects of fluctuations. This was achieved by averaging the oxygen atom positions over a $200 \mathrm{ps}$ for a system of probe radius $R=10 \mathrm{~nm}$ and of film thickness $E=2.2 \mathrm{~nm}$. With this time average, a comparison with the continuous model is possible. Method is the following: first select the molecules above the non-deformed interface, then cut the simulation box in cubes and 
finally count the number of molecules in each cube. The value of the non-deformed interface is obtained by the Gibbs method. The height is obtained from the number of molecules multiplied by the volume of one molecule and divided by the surface of one cell. The size of the box for these simulations is $8 \times 8 \times 8 \mathrm{~nm}^{3}$. The profiles are obtained for distances close to the critical separation distance $D_{\min }$, i.e. right before the occurance of the jump to contact.

\section{Interface fluctuations}

This study aims at evaluating the influence of interface fluctuations on the jump to contact and the surface deformation. Indeed, due to thermal activity water is constantly moving. Its surface fluctuations can generate uncertainties on the evaluation of $D_{\min }$ and $\eta_{\max }$ which are defined using the value of the undistorted interface. Therefore, an evaluation of the fluctuations of both flat and distorded interfaces is made using data obtained from MD simulations using the following method.

A system containing an AFM probe and a 1.8-nm thin film containing 5046 water molecules is constructed in a $8 \times 8 \times 8 \mathrm{~nm}^{3}$ simulation box. This water film is equilibrated at a sufficient distance from the probe to prevent interference with it. Two simulations are then carried to evaluate the water interface fluctuations. In the first one, the probe remains far from the water film so that no interaction is observed. In the second one, the probe is approached at a distance $D_{\min }=12.2 \mathrm{~nm}$ of the water film to obtain a maximum deformation of the interface. Dynamics are then run for $1 \mathrm{~ns}$, the positions of the oxygen atoms being exported every ps i.e. each 1000 timesteps. The simulation box is then divided in cubes of $0.5 \times 0.5 \times 8 \mathrm{~nm}^{3}$, and the number of oxygen atoms in each cube are counted. Following this method a value of the interface height in different points can be obtained at each ps. The standard deviation of the height values thus giving the order of fluctuations of the interface in each cube, and the mean fluctuations of the whole interface. 


\section{Results and discussion}

\section{Parametric study}

The value of the gap between the apex of the probe and the undistorted interface at the moment of the jump to contact, $D_{\min }-R$, and the corresponding deformation height $\eta_{\max }$ were evaluated for each systems. As previously mentionned, the systems modelled belong to the shallow film regime or the intermediate regime. Those two regimes are delimited by a characteristic thickness $E_{\gamma}$ illustrated by the vertical lines on Figure 3.
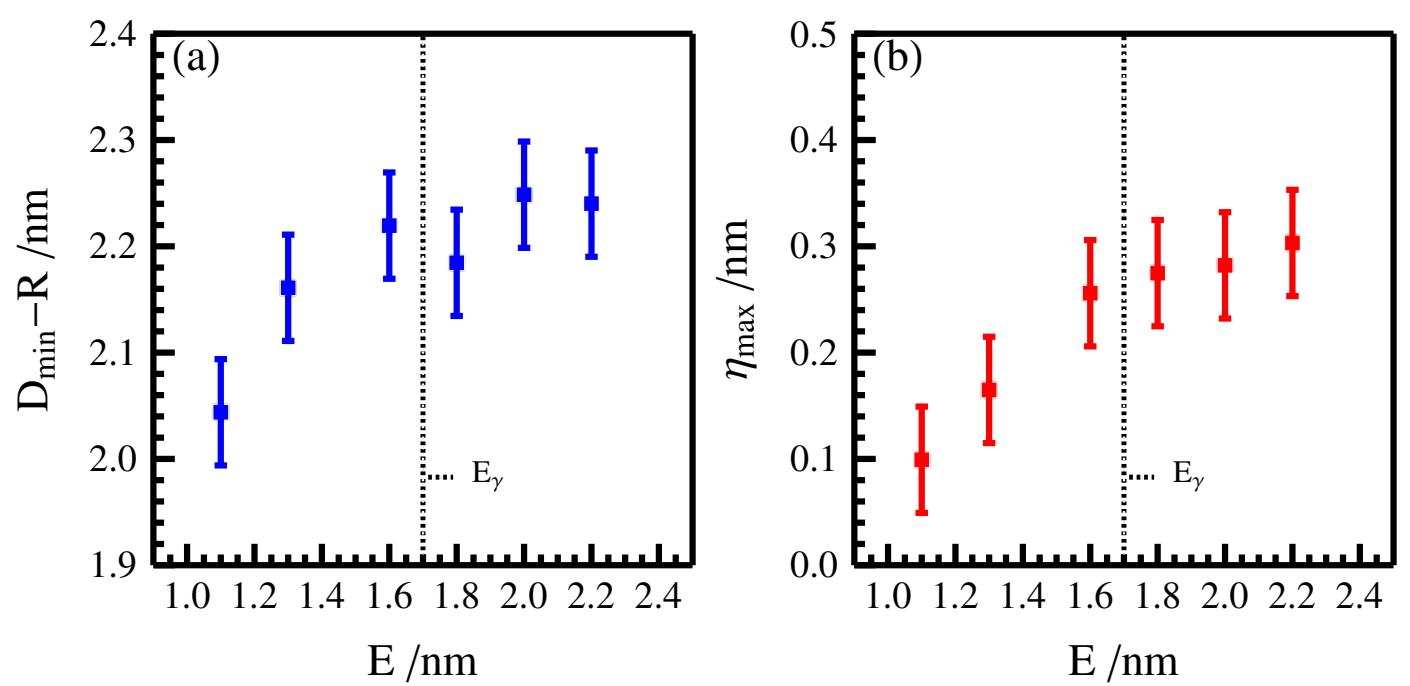

Figure 3: Influence of film thickness on (a) the gap $D_{\min }-R$ and (b) interface deformation $\eta_{\max }$ at the moment of the jump to contact for a system of probe radius $R=10 \mathrm{~nm}$

The influence of liquid thickness $E$ on $D_{\min }-R$ and $\eta_{\max }$ is reported on Figure 3. Results show that for $E<E_{\gamma}, D_{\min }-R$ and $\eta_{\max }$ are increasing with the film thickness. This result is in agreement with the previous findings of Ledesma et al. revealed on the continuous model ${ }^{21}$. For $E>E_{\gamma}, D_{\min }-R$ and $\eta_{\max }$ are also observed to grow with the thickness, but in a less pronounced way. This is explained by the fact that these thicknesses correspond to the beginning of the intermediate regime, in which the increasing of $D_{\min }$ and $\eta_{\max }$ with $E$ becomes less and less pronounced until it reaches a plateau value obtained for 
the characteristic thickness $E_{g}$.
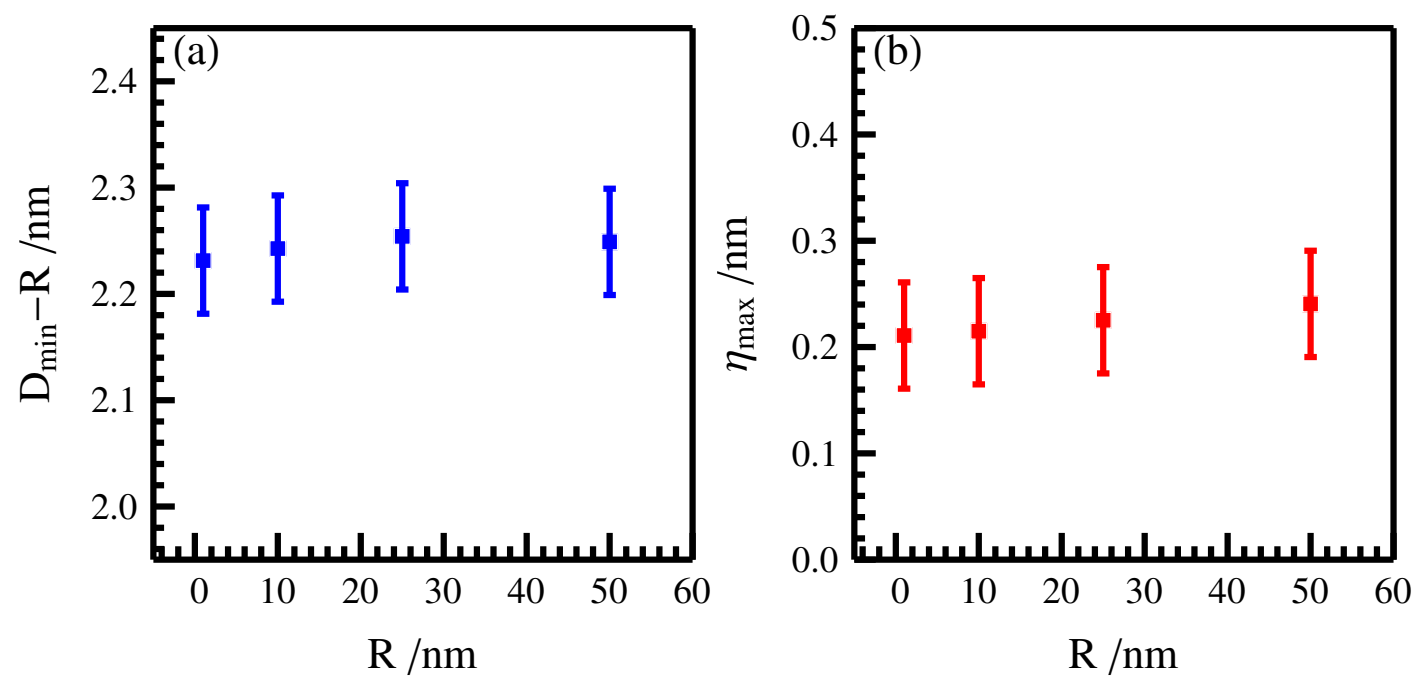

Figure 4: Influence of probe radius on (a) the gap $D_{\min }-R$ and (b) interface deformation $\eta_{\max }$ at the moment of the jump to contact for a system of thickness $E=2.2 \mathrm{~nm}$

The influence of tip radius $R$ on $D_{\min }-R$ and $\eta_{\max }$ are reported on Figure 4 . Results show that the value of $D_{\min }-R$ and $\eta_{\max }$ remain constant, with the considered uncertainty, with an increase of the probe radius. Those results are again consistent with the proposed model for the thin film regime ${ }^{21}$. This is due to the fact that the apex of the deformation sees only the extremity of the probe, which appears to be close to flat for the radii considered.

Hence, most of the findings of the MD model are consistent with the continous model proposed by Ledesma et al.. The observed differences between the obtained values of $D_{\min }-R$ and $\eta_{\max }$ deriving from MD and those resulting from the continuous model are explained by the fluctuations of water. Indeed, the fluctuation of the liquid surface creates an instability of the deformation. The deformation is amplified or flatten by the fluctuations, resulting in an anticipated or retarded jump-to-contact. The values obtained for $\eta_{\max }$ are slightly lower than the theoretical values for systems of similar thickness. Again this is attributed to the fluctuations of the interface which creates an instability of the deformation profile. 
Another difference with the theoretical model is the lack of electrostatic charge on the modelled tip. Indeed, as the tip was assigned a Hamaker potential, only the influence of Van der Waals forces have been considered on the regard of electrostatic forces. Electrostatic forces result from long-range interactions, so it is likely that if the probe was composed of partially charged atoms or molecules it could have interacted more strongly with the liquid, especially if the considered liquid is polar like water. The study of the influence of electrostatic forces on the jump-to-contact phenomenon is an interesting outlook.

\section{Deformation profiles}

Surface deformation is caused by Van der Waals interaction between the probe and the liquid. Molecules at the apex of the deformation are more subject to the probe's attraction and more likely to jump. Once the jump of the molecules occurred, the surface flattens then deforms again. Molecules jump successively on the probe, wetting the latter. Deformation of the liquid surface right before the jump to contact are presented on Figure 5 as a deformation density map and Figure 6 as a 1-D profile. Movies of both surface deformation and jump to contact are available in supplementing material.

The representation on the left of Figure 5 is obtained by averaging the positions of the molecules for 200 ps. The noise observed on the left-map comes from MD data. This density map is radially averaged on the right-map to be compared more easily to the continuous model. The deformation is localized under the apex of the probe, and its height consists of a few water molecules. These results are consistent with the theoretical model as can be seen on Figure 6 where a 1D representation of $\eta$ as a function of $r$ is obtained. This function is compared to the profile resulting from numerical solvation of the modified Laplace equation for the same parameters. 

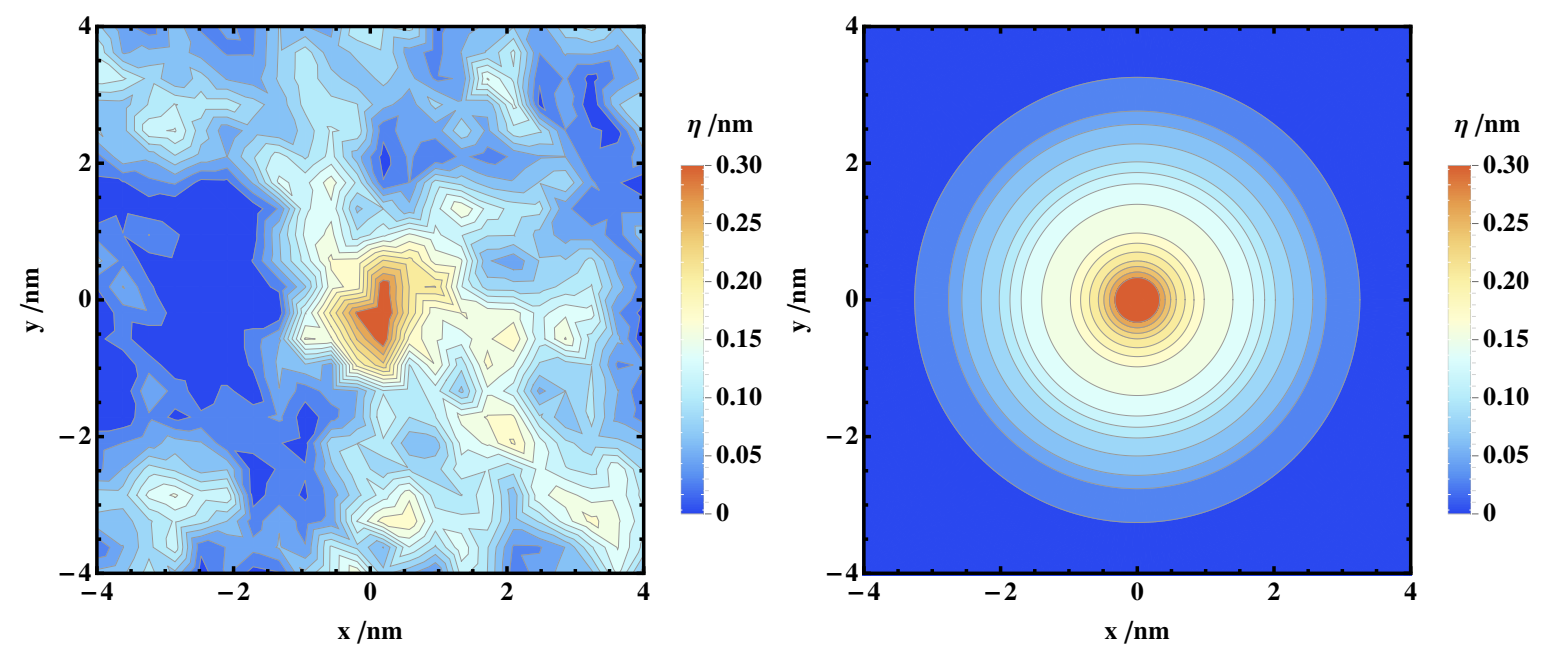

Figure 5: Rough (left) and radially averaged (right) representations of the surface deformation for a system of probe radius $R=10 \mathrm{~nm}$ and of film thickness $E=2.2 \mathrm{~nm}$

The MD profile and the one issued from the continuous model are very similar. The radial extent of both deformation profiles are restricted and situated under the probe for this system. This is consistent with the "localized tip effect" described by the model for the thin film regime. In the MD profile a negative value of $\eta$ is observed on both side of the deformation. This is a box effect explained by the periodic boundary conditions in molecular simulations. Water molecules feeding the deformation comes from the direct surroundings thus resulting in a lack of molecules in that area. Its height remains very small, less than the size of a water molecule, and it is believed that the tendency tends to disappear as the system tends to an equilibrium. MD profiles cannot be averaged over a higher period of time due to the instability caused by interface fluctuations.

\section{Interface fluctuations}

The fluctuation height of the liquid surface was evaluated by analysis of the MD simulations following the previously described method. A map of the fluctuations of the flat interface over $1 \mathrm{~ns}$ is obtained and represented in Figure 7. A map of the fluctuations of the deformed 


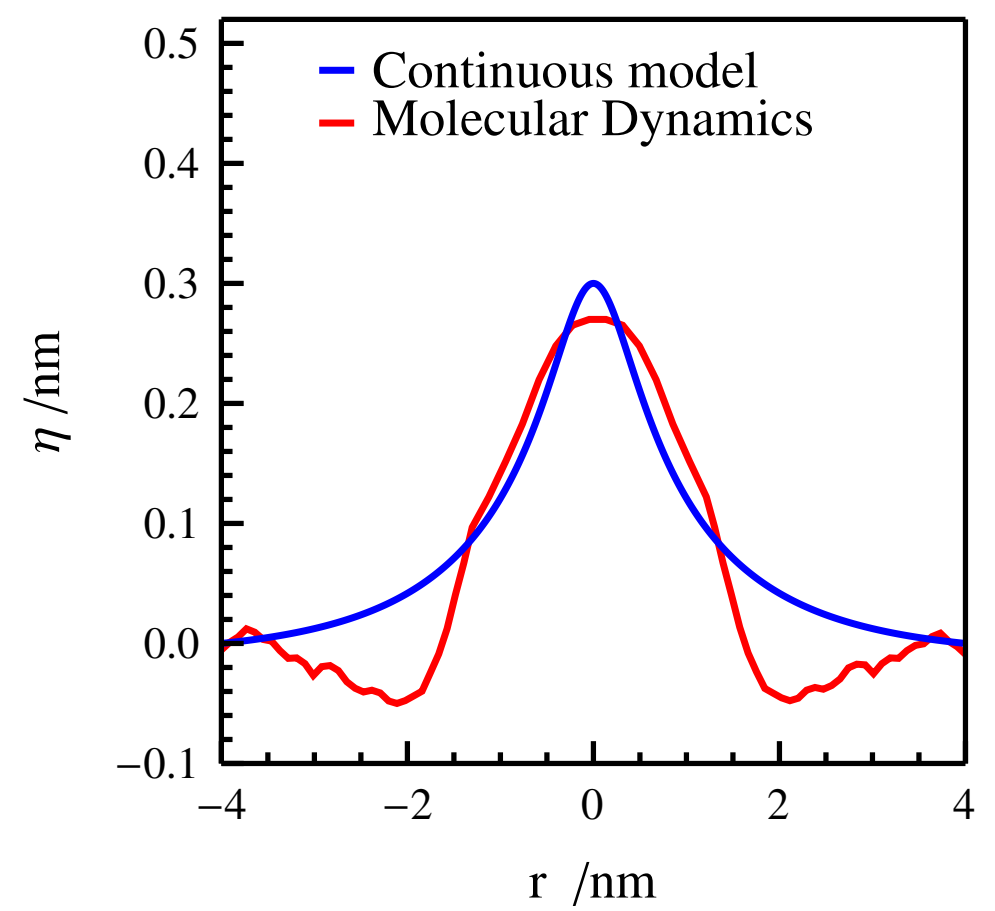

Figure 6: Comparison of MD simulation results (red curve) and theoretical model (blue curve) for a system of probe radius $R=10 \mathrm{~nm}$ and $E=2.2 \mathrm{~nm}$

interface over 1 ns is represented on Figure 8 along with the corresponding rough and radially averaged deformation profiles.

On Figure 7, it is observed that the interface fluctuations are quite homogeneous, differences observed in the map range over $5 \times 10^{-2} \mathrm{~nm}$ which is below the atomic bond length. The average fluctuation of the whole interface is $\sigma_{\mathrm{MD}}=1.47 \times 10^{-1} \mathrm{~nm}$. The capillary wave theory predicts an amplitude of fluctuation of $\sigma_{\text {cap }}=1.9 \times 10^{-1} \mathrm{~nm}$, which is a close value from the one obtained numerically. The capillary wave theory therefore describes well the oscillations of the interface, even at a molecular scale.

On the figure 8, the fluctuations of the deformed interface are represented along with the corresponding interface deformation. The results obtained are similar to those of the flat interface i.e. the fluctuations are homogeneous over the whole interface, and range over less 


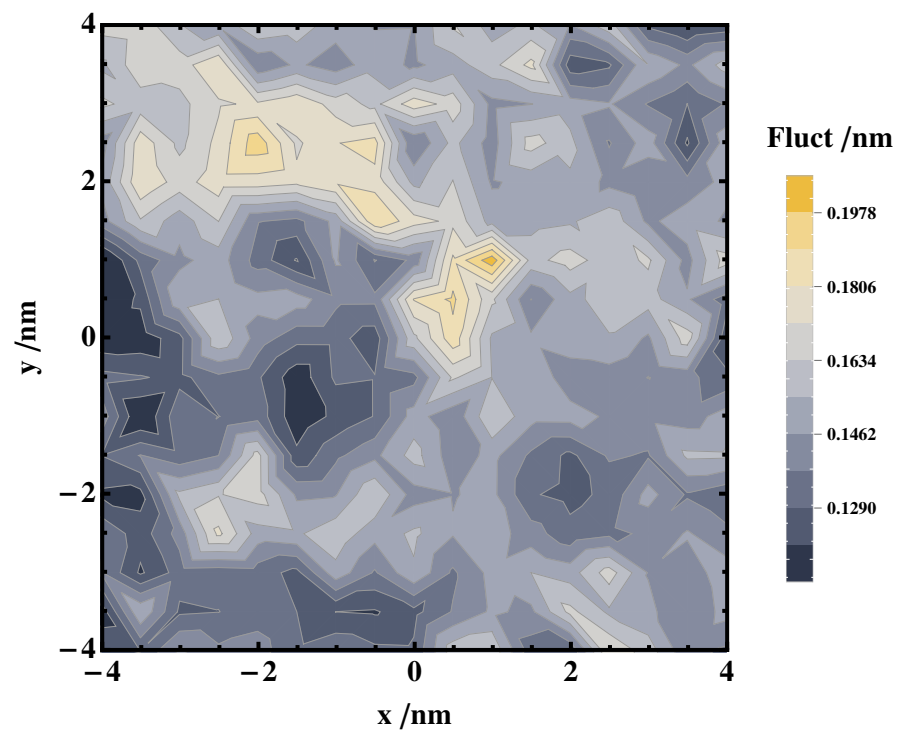

Figure 7: Fluctuations map of a flat interface for a system with $E=1.8 \mathrm{~nm}$ and $R=10 \mathrm{~nm}$ over 1 ns.

than $1 \AA$. It also interesting to point out that the amplitude of the oscillations are independant of the interface deformation. The evolution of fluctuation maps and the corresponding surface deformations over the 1 ns simulation by steps of 25 ps are available as supplementary material. An amplitude of fluctuations of about $1.5 \times 10^{-1} \mathrm{~nm}$ explains the behaviour of the deformation observed in MD simulations. The surface is alternally distorted and flattened due to thermal oscillations. This instability of the deformation can result in an anticipated or postponed jump to contact, which has been observed in the simulations.

\section{Conclusions}

MD simulations have been carried in the thin film regime to evaluate the influence of the liquid film thickness and the probe radius on the distance at which the jump to contact occurs and the corresponding maximum deformation of the liquid surface. Modelled systems belong to the shallow film regime previously described in a continuous model. Results show that, for the thin film regime, the evolution of $\eta_{\max }$ and $D_{\min }-R$ is steady with the increasing 

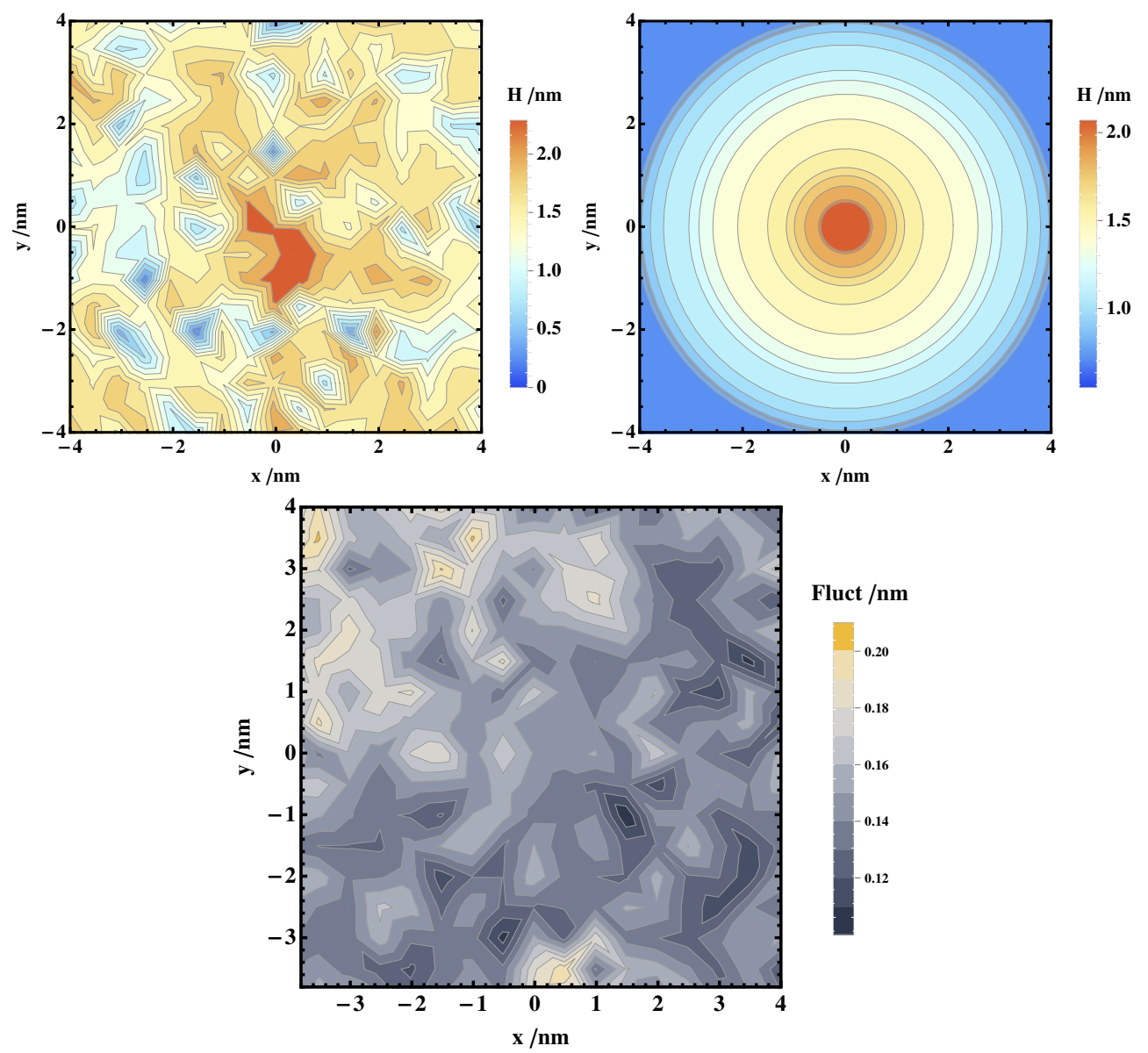

Figure 8: Fluctuations map (bottom) and corresponding deformation profile rough (up left) and radially averaged (up right) of a deformed interface for a system with $E=1.8 \mathrm{~nm}$ and $R=10 \mathrm{~nm}$ over $1 \mathrm{~ns}$.

of film thickness and independent of the probe radius. This evolution is less pronounced when entering the intermediate regime. This parametric study is therefore qualitatively in agreement with the model proposed by Ledesma et al.. However, differences in the values of $D_{\min }-R$ and $\eta_{\max }$ are evidenced due to uncertainties highlighting the influence of the interface fluctuations.

A deformation profile of the liquid surface has also been obtained by averaging the position of water molecules over time. This profile has been compared to the solution of generalized Laplace equation for the considered system and a good agreement is obtained. A small and localized deformation of the order of a few water molecules is observed. This 
deformation is subject to an instability created by the fluctuations of the liquid surface. A further study of the fluctuations shows that the oscillations of the interface are independant of the deformation and have an amplitude which can be described by the capillary wave theory for a flat system.

The MD approach retrieves the behaviour predicted by the continuous model. It points out, however, that for a more accurate description of the phenomenon occurring at nanoscale, fluctuations have to be taken in consideration in the models. Recent theoretical studies $^{34}$ showed that coalescence is controled by initial fluctuating regime which depends of capillary fluctuations of the interface. The fact that these fluctuations do not depend on the curvature or on the external force facilitates a predictive macroscopic theory of coalescence that takes into account the initial role of fluctuations in a consistent way.

\section{Acknowledgement}

The author thanks Magali DUVAIL and Frédéric RISSO for constructive discussions. The author also thanks CEA for the amont-aval thesis financial support, and Marcoule's computing center for high computing facilities. This work was also granted access to the HPC resources of [TGCC/CINES/IDRIS] under the allocation 2019-A0070911087 made by GENCI (Grand Equipement National de Calcul Intensif).

\section{Supporting Information Available}

The following files are available free of charge.

- surface_deformation.mpg: Film of the surface deformation obtained in MD (500 ps simulation) for a system composed of a $10 \mathrm{~nm}$ probe and a $2.2 \mathrm{~nm}$ water film.

- jump_to_contact.mpg: Film of the jump to contact obtained in MD (1 ns simulation) 
for a system composed of a $10 \mathrm{~nm}$ probe and a $2.2 \mathrm{~nm}$ water film.

- fluctuations_deformed_interface.avi: Evolution of the fluctuation map for a distorted interface (1 ns simulation) for a system composed of a $10 \mathrm{~nm}$ probe and a $1.8 \mathrm{~nm}$ water film.

- deformation_profiles.avi: Evolution of the interface deformation corresponding to the previous evolution of fluctuation maps.

This material is available free of charge via the Internet at http://pubs.acs.org/.

\section{Bibliography}

(1) Butt, H. J.; Cappella, B.; Kappl, M. Force Measurements with the Atomic Force Microscope: Technique, Interpretation and Applications. Surface Science Reports 2005, $59,1-152$.

(2) Binnig, G.; Quate, C. F.; Gerber, C. Atomic Force Microscope. Physical Review Letters 1986, 56, 930 .

(3) Pompe, T.; Herminghaus, S. Three-Phase Contact Line Energetics from Nanoscale Liquid Surface Topographies. Physical Review Letters 2000, 85, 1930-1933.

(4) Fery, A.; Pompe, T.; Herminghaus, S. Nanometer Resolution of Liquid Surface Topography by Scanning Force Microscopy. Journal of Adhesion Science and Technology 1999, 13, 1071-1083.

(5) Checco, A.; Guenoun, P.; Daillant, J. Nonlinear Dependence of the Contact Angle of Nanodroplets on Contact Line Curvature. Physical Review Letters 2003, 91, 186101.

(6) Pompe, T.; Fery, A.; Herminghaus, S. Imaging Liquid Structures on Inhomogeneous Surfaces by Scanning Force Microscopy. Langmuir 1998, 14, 2585-2588. 
(7) Dubreuil, F.; Elsner, N.; Fery, A. Elastic Properties of Polyelectrolyte Capsules Studied by Atomic-Force Microscopy and RICM. Eur. Phys. J. E 2003, 12, 215-221.

(8) Berg, J. K.; Weber, C. M.; Riegler, H. Impact of Negative Line Tension on the Shape of Nanometer-Size Sessile Droplets. Physical Review Letters 2010, 105, 076103.

(9) Sutton, A. P.; Todorov, T. N. Mechanical and Electrical Properties of Metallic Contacts at the Nanometer Scale. Journal of Physics and Chemistry of Solids 1994, 55, 11691174.

(10) Wu, J.-J. The Jump-to-Contact Distance in Atomic Force Microscopy Measurement. The Journal of Adhesion 2010, 86, 1071-1085.

(11) Kuipers, L.; Frenken, J. W. M. Jump to Contact, Neck Formation, and Surface Melting in the Scanning Tunneling Microscope. Physical Review Letters 1993, 70, 3907-3910.

(12) Checco, A.; Schollmeyer, H.; Daillant, J.; Guenoun, P.; Boukherroub, R. Nanoscale Wettability of Self-Assembled Monolayers Investigated by Noncontact Atomic Force Microscopy. Langmuir 2006, 22, 116-126.

(13) Cappella, B.; Dietler, G. Force-Distance Curves by Atomic Force Microscopy. Surface Science Reports 1999, 34, 104.

(14) Ledesma-Alonso, R.; Tordjeman, P.; Legendre, D. Multiscale Deformation of a Liquid Surface in Interaction with a Nanoprobe. Physical Review E 2012, 85, 1-10.

(15) Bowen, J.; Cheneler, D. A Dynamic Model of the Jump-To-Contact Phenomenon During AFM Analysis. Langmuir 2012, 28, 17273-17286.

(16) Ledesma-Alonso, R.; Legendre, D.; Tordjeman, P. Nanoscale Deformation of a Liquid Surface. Physical Review Letters 2012, 108, 1-5.

(17) Israelachvili, J. Intermolecular and Surface Forces; 2011. 
(18) Schatz, G. C. Using Theory and Computation to Model Nanoscale Properties. PNAS 2007, 104, 6885-6892.

(19) Landman, U.; Luedtke, W. D.; Burnham, N. A.; Colton, R. J. Atomistic Mechanisms and Dynamics of Adhesion, Nanoindentation, and Fracture. Science 1990, 248, 454.

(20) Bickel, T. Probing nanoscale deformations of a fluctuating interface. EPL 2014, 106, 16004 .

(21) Ledesma-Alonso, R.; Legendre, D.; Tordjeman, P. AFM Tip Effect on a Thin Liquid Film. Langmuir 2013, 29, 7749-7757.

(22) Hamaker, H. C. The London-Van der Waals Attraction Between Spherical Particles. Physica IV 1937, 10, 1058.

(23) Barrat, J.-L.; Hansen, J.-P. Basic Concepts for Simple and Complex Liquids; 2003.

(24) Ledesma-Alonso, R.; Mortagne, C.; Chireux, V.; Ledesma-Alonso, R.; Ogier, M.; Risso, F.; Ondarçuhu, T.; Legendre, D.; Tordjeman, P. Near-field deformation of a liquid interface by atomic force microscopy. microscopy. Physical Review E 2017, 96, $12802-1$.

(25) MacDowell, L. G. Capillary wave theory of adsorbed liquid films and the structure of the liquid-vapor interface. 2017, 96 .

(26) Liu, H.; Cao, G. Effectiveness of the Young-Laplace Equation at Nanoscale. Scientific Reports 2016, 6, 1-10.

(27) Plimpton, S. Fast Parallel Algorithms for Short-Range Molecular Dynamics. Journal of Computational Physics 1995, 117, 1-19.

(28) Mark, P.; Nilsson, L. Structure and Dynamics of the TIP3P, SPC, and SPC/E Water Models at 298 K. J. Phys. Chem A 2001, 105, 9955. 
(29) Ryckaert, J.-P.; Ciccotti, G.; Berendsen, H. J. C. Numerical Integration of the Cartesian Equations of Motion of a System with Constraints: Molecular Dynamics of n-Alkanes. Journal of Computational Physics 1977, 23, 321-341.

(30) Berendsen, H. J. C. The Missing Term in Effective Pair Potentials. J. Phys. Chem 1987, 91, 6269-6271.

(31) Insight into the molecular mechanism of water evaporation via the finite temperature string method. J. Chem. Phys 2013, 138, 134707.

(32) Smith, P. E.; van Gunsteren, W. F. The viscosity of SPC and SPC/E water at 277 and 300 K. Chemical Physics Letters 1993, 215, 315-318.

(33) Vega, C. Surface tension of the most popular models of water by using the test-area simulation method. J. Chem. Phys 2007, 126, 154707.

(34) Perumanath, S.; Borg, M. K.; Chubynsky, M. V.; Sprittles, J. E.; Reese, J. M. Droplet Coalescence is Initiated by Thermal Motion. Physical Review Letters 2019, 122, 104501. 